\title{
A confirmation of agreement of different approaches for scalar gauge-invariant metric perturbations during inflation
}

\author{
1,2 Mariano Anabitarte* and ${ }^{1,2}$ Mauricio Bellini ${ }^{\dagger}$ \\ ${ }^{1}$ Departamento de Física, Facultad de Ciencias Exactas y Naturales, \\ Universidad Nacional de Mar del Plata, \\ Funes 3350, (7600) Mar del Plata, Argentina. \\ ${ }^{2}$ Consejo Nacional de Investigaciones Científicas y Técnicas (CONICET).
}

\begin{abstract}
We revisit an extension of the well-known formalism for gauge-invariant scalar metric fluctuations, to study the spectrums for both, the inflaton and gauge invariant (scalar) metric fluctuations in the framework of a single field inflationary model where the quasi-exponential expansion is driven by an inflation which is minimally coupled to gravity. The proposal here examined is valid also for fluctuations with large amplitude, but for cosmological scales, where vector and tensor perturbations can be neglected and the fluid is irrotacional.
\end{abstract}

\footnotetext{
* E-mail address: anabitar@mdp.edu.ar

$\dagger$ E-mail address: mbellini@mdp.edu.ar
} 


\section{INTRODUCTION}

The big bang theory has provided a remarkably successful description of the evolution of the universe and now rests upon solid observational foundations. Over the last decades cosmologists have also drawn up architectural sketches of a model which may account for the origin and evolution of structure within this framework: primordial perturbations from an early epoch of inflation, which subsequently grow by gravitational instability to form galaxies and larger structures. The standard picture of inflation introduced in 1981[1] relied on a scalar field, called the inflaton, which during inflation was assumed to have no interaction with any other field. The inflationary scenario postulates that the universe underwent a phase of very rapid, accelerated expansion in its distant past. Observations have provided strong support for the paradigm. However, despite this success, the mechanism which drove the inflationary expansion has yet to be indentified. A multitude of inflationary models involving a broad range of energy scales have been discussed in the literature, including chaotic inflation [2], warm inflation[3], stochastic inflation[4], fresh inflation[5], brane inflation[6], STM inflation[7], and many others.

The scalar fluctuations of the metric are associated with density perturbations. These are the spin-zero projections of metric perturbations and were induced by the vacuum fluctuations of the inflaton field during inflation. Furthermore, they played a crucial role in the generation of primordial inhomogeneities which gave rise to the large scale structure of the present day universe as well as the observed anisotropies of the cosmic microwave background of radiation. Gauge invariance guarantees that equation for the fluctuations of the geometry do not change when moving from one coordinate system to other. This allows us to formulate the problem of the evolution for the amplitude of scalar metric perturbations around the Friedmann-Robertson-Walker (FRW) universe in a coordinate-independent manner at every moment in time. The issue of gauge invariance becomes critical when attempt to analyze how the scalar metric perturbations produced in the early universe influence a globally flat, isotropic and homogeneous universe on super Hubble scales. Space-time fluctuations can also lead to decoherence of matter waves[10]. In the infrared (IR) sector these fluctuations can be represented by a coarse-grained field, which describes a stochastic dynamics[11]. With respect to perturbative approaches, second order gauge invariant perturbations quantities have been calculated in [12] and third order perturbations are supposed to be negligible[13] 
during inflation, but not for large fluctuations.

In this work we examine a perturbed FRW metric using the proposal developed in [14], which is an extension of the well known linearized line element $d s^{2}=(1+2 \psi) d t^{2}-a(t)^{2}(1-$ $2 \psi) d \vec{r}^{2}$, for a longitudinal gauge. Our results show a coincidence of different approaches for small fluctuations; the formalism developed in [14] and the standard method.

\section{FORMALISM}

We consider a scalar field $\varphi$ which is minimally coupled to gravity. The action of the system is

$$
I=\int d^{4} x \sqrt{-g}\left[\frac{\mathcal{R}}{16 \pi G}+\frac{1}{2} g^{\mu \nu} \varphi_{, \mu} \varphi_{, \nu}-V(\varphi)\right],
$$

where $g=-e^{-4 \psi} a^{6}(t)$ is the determinant of the covariant metric tensor with components $g_{\mu \nu}(\mu, \nu$ run from 0 to 3$)$ and $V(\varphi)$ is the potencial related to the inflaton field. To study the gauge invariant scalar metric fluctuations $\psi\left(x^{\alpha}\right)$, we propose the following perturbed metric

$$
d S^{2}=e^{2 \psi} d t^{2}-a^{2}(t) e^{-2 \psi} d \vec{r}^{2}
$$

where $d \vec{r}^{2}=d x^{2}+d y^{2}+d z^{2}$ and $\psi(t, \vec{r})$ is the scalar metric fluctuation. The metric (2) is the perturbed version of the background FRW one, which is spatially flat, isotropic, homogeneous and has a scalar curvature $\overline{\mathcal{R}}=6\left[\frac{\ddot{a}}{a}+\left(\frac{\dot{a}}{a}\right)^{2}\right]$. This metric describe nonperturbative gravitational fluctuations on cosmological scales, on which vector and tensor perturbations of the metric can be neglected and the fluid can be considered as irrotacional. Furthermore, using the continuity equation on large scales

$$
\frac{\partial \rho}{\partial \tau}=-3 \mathcal{H}(\rho+P)
$$

where $\mathcal{H}=\frac{d}{d \tau}\left[\ln \left(a(t) e^{-\psi}\right)\right]$ and $d \tau=e^{\psi} d t$. One can show that exists a conserved quantity in time at any order in perturbation theory

$$
f=\ln \left(a e^{-\psi}\right)+\frac{1}{3} \int^{\rho} \frac{d \rho^{\prime}}{\left(P^{\prime}+\rho^{\prime}\right)} .
$$

Considering the invariant $\omega$, which characterizes the equation of state $P=\omega \rho$. The perturbation $\delta f=-\psi+\frac{1}{3(1+\omega)} \ln (\rho / \bar{\rho})$, is a gauge-invariant quantity representing the non-linear extension of the curvature perturbation for adiabatic fluids on uniform energy density hypersurfaces on superhorizon scales[14]. Here, $(\bar{P}, \bar{\rho})$ denote the background pressure and 
energy density and $(P, \rho)$ denote pressure and energy density on the perturbed metric (2). The scalar curvature of this metric is

$$
\mathcal{R}=6 e^{-2 \psi}\left[\left[\frac{\ddot{a}}{a}+\left(\frac{\dot{a}}{a}\right)^{2}\right]-\ddot{\psi} e^{4 \psi}-5 \dot{\psi}\left(\frac{\dot{a}}{a}\right)+\frac{e^{4 \psi}}{3 a^{2}}\left[\nabla^{2} \psi-(\vec{\nabla} \psi)^{2}\right]+3 \dot{\psi}^{2}\right] .
$$

Now we shall use the Lagrangian formalism to describe the dynamics of $\varphi$ and $\psi$. The equation of motion for $\varphi$ is

$$
\ddot{\varphi}+\left[3\left(\frac{\dot{a}}{a}\right)-4 \dot{\psi}\right] \dot{\varphi}-\frac{1}{a^{2}} e^{4 \psi} \nabla^{2} \varphi+e^{2 \psi} V^{\prime}(\varphi)=0
$$

where $V^{\prime}(\varphi) \equiv \frac{d V}{d \varphi}$. The equation (6) is an operatorial one because $\varphi$ is considered as a quantum scalar field. The equation of motion for $\psi$ is

$$
\left[\frac{\partial \mathcal{R}}{\partial \psi}-\frac{\partial}{\partial x^{\mu}} \frac{\partial \mathcal{R}}{\partial \psi_{, \mu}}\right]-2 \mathcal{R}-\frac{1}{\sqrt{-g}} \frac{\partial \sqrt{-g}}{\partial t} \frac{\partial \mathcal{R}}{\partial \dot{\psi}}=32 \pi G\left[e^{-2 \psi} \dot{\varphi}^{2}-e^{2 \psi} \frac{1}{a^{2}}(\vec{\nabla} \varphi)^{2}-V(\varphi)\right],
$$

such that both, $\varphi$ and $\psi$ comply with the commutation relations

$$
\begin{aligned}
& {\left[\varphi(t, \vec{r}), \Pi_{\varphi}(t, \vec{r})\right]=i \delta^{(3)}(\vec{r}-\vec{r}),} \\
& {\left[\psi(t, \vec{r}), \Pi_{\psi}(t, \vec{r})\right]=i \delta^{(3)}(\vec{r}-\vec{r}),}
\end{aligned}
$$

$\Pi_{\varphi}$ and $\Pi_{\psi}$ being respectively the conjugate momentums for $\varphi$ and $\psi$ :

$$
\Pi_{\varphi}=\frac{\partial L}{\partial \dot{\varphi}}, \quad \Pi_{\psi}=\frac{\partial L}{\partial \dot{\psi}},
$$

such that $L=\sqrt{-g}\left[\frac{\mathcal{R}}{16 \pi G}+\frac{1}{2} g^{\mu \nu} \varphi_{, \mu} \varphi_{, \nu}-V(\varphi)\right]$ is the density Lagrangian of the system. To complete the description of the dynamics, we need to write the Einstein equations $G_{\alpha \beta}=$ $-8 \pi G T_{\alpha \beta}$. Taking into account cartesian coordinates, the diagonal Einstein equations are

$$
\begin{aligned}
& -\frac{2}{a^{2}} e^{4 \psi} \nabla^{2} \psi+6 H \dot{\psi}-3 \dot{\psi}^{2}+\frac{e^{4 \psi}}{a^{2}}(\vec{\nabla} \psi)^{2}+3 H^{2} \\
& =-8 \pi G\left[\frac{\dot{\varphi}^{2}}{2}+e^{4 \psi} \frac{1}{2 a^{2}}(\vec{\nabla} \varphi)^{2}+V(\varphi) e^{2 \psi}\right] \\
& -8 \pi G\left[\frac{3 e^{-4 \psi}}{2} \dot{\varphi}^{2}-\frac{1}{2 a^{2}}(\vec{\nabla} \varphi)^{2}-3 e^{-2 \psi} V\right]=-\frac{2}{a^{2}}(\vec{\nabla} \psi)^{2}-24 H \dot{\psi} e^{-4 \psi} \\
& +15 \dot{\psi}^{2} e^{-4 \psi}+6 e^{-4 \psi}\left(\frac{\ddot{a}}{a}\right)-6 \ddot{\psi} e^{-4 \psi}-3 H^{2} e^{-4 \psi}
\end{aligned}
$$

where $G_{\beta}^{\alpha}=R_{\beta}^{\alpha}-\frac{1}{2} \mathcal{R} g_{\beta}^{\alpha}$ is the Einstein tensor and $T_{\beta}^{\alpha}=\varphi^{, \alpha} \varphi_{, \beta}-g_{\beta}^{\alpha}\left[\frac{1}{2} \varphi^{, \rho} \varphi_{, \rho}-V(\varphi)\right]$ is the Energy - Momentum tensor for a scalar field. On the other hand, the non-diagonal Einstein equations have the form

$$
\frac{1}{a} \frac{\partial}{\partial x^{i}}\left[\frac{\partial}{\partial t}(a \psi)\right]-\frac{\partial \psi}{\partial t} \frac{\partial \psi}{\partial x^{i}}=4 \pi G \frac{\partial \varphi}{\partial t} \frac{\partial \varphi}{\partial x^{i}}
$$


so that, using the eq. (12) in eqs. (10) and (11), we obtain the exact equation of motion for $\psi$

$$
\ddot{\psi}+7 H \dot{\psi}-\frac{e^{4 \psi}}{a^{2}} \nabla^{2} \psi-4 \dot{\psi}^{2}-\frac{5 e^{4 \psi}}{3 a^{2}}(\vec{\nabla} \psi)^{2}+8 \pi G V(\varphi) e^{2 \psi}=-\frac{8 \pi G}{3 a^{2}}(\vec{\nabla} \varphi)^{2}
$$

which describes the dynamics of $\psi$ with arbitrary amplitude. However, it is very difficult to solve these equations in an exact manner. Notice that we have used the Einstein equations, and not the Lagrange one, to obtain the dynamics of $\psi$. One could make the inverse procedure, because both manners to work are equivalent. However, in this case the calculations with the Einstein equations are more simple.

\section{LINEAR APPROXIMATION}

In the weak field limit, it is sufficient to make a linear approximation on the scalar metric perturbations, so that one can write $e^{ \pm 2 \psi\left(x^{\alpha}\right)} \simeq 1 \pm 2 \psi\left(x^{\alpha}\right)$ in the exact equations of motion (6) and (13), for $\psi$ and $\varphi$. In this limit the metric (2) preserves gauge invariance and the linearized line element

$$
d S^{2}=(1+2 \psi) d t^{2}-a(t)^{2}(1-2 \psi) d \vec{r}^{2}
$$

takes the form of a longitudinal gauge so that coordinate transformations induce difeomorfism transformations [8].

The equation of motion for the inflaton in its exact form is given by (6). However, in the weak field limit we can make the semi-classical approximation $\varphi\left(x^{\alpha}\right)=\langle E|\varphi| E\rangle+\phi\left(x^{\alpha}\right)$. Here, $\langle E|\varphi| E\rangle=\phi_{c}(t)$ is the expectation value of $\varphi$ evaluated on the quantum state $|E\rangle$. Furthermore, in this limit the quantum fluctuations of the inflaton field are considered to be very small and $\langle E|\phi| E\rangle=\langle E|\dot{\phi}| E\rangle=0$. With this representation we obtain the following dynamical equations for the fluctuations of the inflaton field $\phi$ and the classical field $\phi_{c}$ :

$$
\begin{aligned}
& \ddot{\phi}+3\left(\frac{\dot{a}}{a}\right) \dot{\phi}-\frac{1}{a^{2}} \nabla^{2} \phi+V^{\prime \prime}\left(\phi_{c}\right) \phi=4 \dot{\psi} \dot{\phi}_{c}+2 \psi V^{\prime}\left(\phi_{c}\right), \\
& \ddot{\phi}_{c}+3\left(\frac{\dot{a}}{a}\right) \dot{\phi}_{c}+V^{\prime}\left(\phi_{c}\right)=0 .
\end{aligned}
$$

The background Friedmann equations are

$$
3 H_{c}^{2}=8 \pi G\left[\frac{\dot{\phi}_{c}^{2}}{2}+V\left(\phi_{c}\right)\right]
$$


where $\left.V\left(\phi_{c}\right) \equiv V(\varphi)\right|_{\phi_{c}}$ is the scalar potential evaluated on the classical background field $\phi_{c}(t)$ and the Hubble parameter with back-reaction effects included is

$$
H=\frac{\dot{a}}{a} \simeq H_{c}(t)+\frac{4 \pi G}{3 H_{c}}\left\langle E\left|\frac{\dot{\phi}^{2}}{2}+\frac{(\vec{\nabla} \phi)^{2}}{2 a^{2}}+\sum_{n=1}^{\infty} \frac{V^{(n)}\left(\phi_{c}\right)}{n !} \phi^{n}\left(x^{\alpha}\right)\right| E\right\rangle .
$$

When the metric fluctuations are small it is sufficient to make $H_{c} \simeq \frac{\dot{a}}{a}$, because the last term in the right hand of the expression (17) is negligible with respect to the first one. This approximation is valid only on large scales, which are super Hubble scales during the inflationary epoch. Furthermore, the primer denotes the derivative with respect to $\varphi$, such that $\left.V^{\prime}\left(\phi_{c}\right) \equiv \frac{d V(\varphi)}{d \varphi}\right|_{\phi_{c}}$.

Furthermore, the Einstein equations (12) now hold

$$
\frac{1}{a} \frac{\partial^{2}}{\partial x^{i} \partial t}[a \psi]=4 \pi G \frac{\partial}{\partial x^{i}}\left[\dot{\phi}_{c} \phi\right]
$$

from which (once we have taken into account that $\langle E|\phi| E\rangle=0$ ) we obtain that the evolution for the expectation value of $\psi$ goes as

$$
\langle E|\psi| E\rangle \sim a^{-1}
$$

which decreases with the inverse of the scale factor of the universe. Finally, the linearized dynamics of $\psi$ can be obtained from the Einstein equations (10) and (11): $\delta G^{\mu}{ }_{\nu}=-8 \pi G \delta T_{\nu}^{\mu}$

$$
\ddot{\psi}+\left[H-2 \frac{\ddot{\phi}_{c}}{\dot{\phi}_{c}}\right] \dot{\psi}-\frac{1}{a^{2}} \nabla^{2} \psi+2\left[\dot{H}-\frac{\ddot{\phi}_{c}}{\dot{\phi}_{c}} H\right] \psi=0
$$

which, as one expects, is the same as the equation obtained in[9]. Note that the equation (20) is the equation (13) once linearized, with the constriction (18).

\section{AN EXAMPLE}

In this section we shall illustrate the formalism in the linearized approximation, when the expansion is governed by a power-law expansion $a=\beta t^{p}$. In this case the Hubble parameter is given by $H=p / t$ and the classical field $\phi_{c}(t)$ is

$$
\phi_{c}(t)=\phi_{0}\left[1-\ln \left(\frac{H_{0} t}{p}\right)\right]
$$

where the power $p$ is

$$
p=4 \pi G \phi_{0}^{2}
$$


$\phi_{0}$ being the value of $\phi_{c}\left(t_{0}\right)$ when inflation begins (i.e., at $\left.t=t_{0}\right)$. The equation of state is given by $\omega=\frac{\bar{P}}{\bar{\rho}}=\frac{-(p-2 / 3)}{p}, \omega$ being an invariant. Furthermore, the classical potential $V\left(\phi_{c}\right)$ is

$$
V\left(\phi_{c}\right)=\frac{3 H_{0}^{2}}{8 \pi G}\left(\frac{3 p-1}{3 p}\right) e^{2 \phi_{c} / \phi_{0}} .
$$

The solution for the $\psi$-modes, once normalized, are

$$
\xi_{k}(t)=\frac{\sqrt{\pi}}{2} \sqrt{\frac{t}{(p-1)}} \mathcal{H}_{\mu}^{(2)}\left[\frac{k t^{1-p}}{(p-1) \beta}\right] \times\left(\frac{t}{t_{0}}\right)^{-(p+2) / 2},
$$

where $\mathcal{H}_{\mu}^{(2)}[x(t)]$ is the second kind Hankel function with $\mu=\frac{p+1}{2(p-1)}$. Using the small argument Hankel functions limit, we obtain that these modes have the following expression on super Hubble scales:

$$
\left.\xi_{k}(t)\right|_{k \gg 1 /(a H)} \simeq i \sqrt{\frac{\pi}{4(p-1)}} \Gamma\left[\frac{(p+1)}{2(p-1)}\right]\left[\frac{2(p-1) \beta}{\pi}\right]^{\frac{(p+1)}{2(p-1)}} k^{\frac{-(p+1)}{2(p-1)}}
$$

which is independent of time. The equation of motion for the modes of the inflaton field on cosmological scales can be approximated to

$$
\begin{aligned}
& \ddot{\tilde{\xi}}_{k}(t)+\frac{3 p}{t} \dot{\tilde{\xi}}_{k}(t)+\left[\frac{k^{2}}{\beta^{2} t^{2 p}}+\frac{4\left(6 \pi G \phi_{0}^{2}-1\right)}{t^{2}}\right] \tilde{\xi}_{k}(t) \\
& =\mathrm{i}\left[\frac{(3 p-2) \phi_{0}}{t^{2}}\right] \sqrt{\frac{\pi}{p-1}} \Gamma\left(\frac{p+1}{2(p-1)}\right)\left[\frac{2 \beta(p-1)}{\pi}\right]^{\frac{(p+1)}{2(p-1)}} k^{-\frac{(p+1)}{2(p-1)}},
\end{aligned}
$$

which, on super Hubble scales, has the solution

$$
\begin{aligned}
& \tilde{\xi}_{k}(t) \simeq t^{\frac{1}{2}(1-3 p)} \\
& \times\left\{A \mathcal{J}_{\nu_{2}}[x(k, t)]+B \mathcal{Y}_{\nu_{2}}[x(k, t)]-\mathrm{i} \gamma \beta \sqrt{\frac{\pi}{p-1}} \Gamma\left(\frac{p+1}{2(p-1)}\right)\left[\frac{2 \beta(p-1)}{\pi}\right]^{\frac{p+1}{2(p-1)}} k^{\frac{1-3 p}{2(p-1)}}\right. \\
& \times\left[\mathcal{J}_{\nu_{2}}[x(k, t)] \int \frac{d t t^{\frac{5}{2}(p-1)} \mathcal{Y}_{\nu_{2}}[x(k, t)]}{\left[\mathcal{J}_{\nu_{2}}[x(k, t)] \mathcal{Y}_{\nu_{1}}[x(k, t)]-\mathcal{Y}_{\nu_{2}}[x(k, t)] \mathcal{J}_{\nu_{1}}[x(k, t)]\right]}\right. \\
&- {\left.\left.\left[\mathcal{Y}_{\nu_{2}}[x(k, t)] \int \frac{d t t^{\frac{5}{2}(p-1)} \mathcal{J}_{\nu_{2}}[x(k, t)]}{\left[\mathcal{J}_{\nu_{2}}[x(k, t)] \mathcal{Y}_{\nu_{1}}[x(k, t)] \mathcal{Y}_{\nu_{2}}[x(k, t)] \mathcal{J}_{\nu_{1}}[x(k, t)]\right]}\right]\right]\right\} }
\end{aligned}
$$


where $\gamma=3(p-2) \phi_{0}, 3 p>m^{2}=4\left(6 \pi G \phi_{0}^{2}-1\right), \nu_{2}=\frac{\sqrt{9 p^{2}-6 p+1-4 m^{2}}}{2(p-1)}, \nu_{1}=1-\nu_{2}$ and $x(k, t)=\frac{k t^{(1-p)}}{\beta(p-1)}$. For $x(t) \ll 1$ this solution can be written as

$$
\begin{aligned}
\tilde{\xi}_{k}(t) & \simeq-\frac{B}{\pi} \Gamma\left(\nu_{2}\right)\left(\frac{k}{2 \beta(p-1)}\right)^{-\nu_{2}} t^{-(1-p) \nu_{2}+\frac{1}{2}(1-3 p)} \\
& +\mathrm{i} \beta(3 p-2) \phi_{0} \Gamma\left(\frac{p+1}{2(p-1)}\right)\left[\frac{2 \beta(p-1)}{\pi}\right]^{\frac{p+1}{2(p-1)}} \\
& \times\left[\frac{\Gamma\left(\nu_{2}+1\right) t^{-\left(\nu_{1}+1\right)(1-p)}}{2 \Gamma\left(\nu_{1}+1\right)\left[\left(\nu_{2}-\nu_{1}\right)(p-1)\right]}\left(\frac{k}{2 \beta(p-1)}\right)^{\nu_{2}-\nu_{1}}\right. \\
& \times \sum_{n=0}^{\infty} \frac{1}{\left[\frac{5 p-3+2 \nu_{1}(p-1)}{4\left(\nu_{2}-\nu_{1}\right)(1-p)}+1\right]}\left[-\frac{\Gamma\left(\nu_{2}+1\right) \Gamma\left(\nu_{1}\right)}{\Gamma\left(\nu_{2}\right) \Gamma\left(\nu_{1}+1\right)} x(k, t)\right]^{2 n\left(\nu_{2}-\nu_{1}\right)} \\
& +\frac{\Gamma\left(\nu_{2}\right) t^{-\left(2 \nu_{2}+1\right)(1-p)}}{2 \Gamma\left(\nu_{2}+1\right)\left(\nu_{2}-\nu_{1}\right)(p-1)}\left(\frac{k}{2 \beta(p-1)}\right)^{-2 \nu_{2}} \\
& \left.\times \sum_{n=0}^{\infty} \frac{1}{\left[\frac{5 p-3+2 \nu_{2}(p-1)}{4\left(\nu_{2}-\nu_{1}\right)(p-1)}+1\right]}\left[-\frac{\Gamma\left(\nu_{1}+1\right) \Gamma\left(\nu_{2}\right)}{\Gamma\left(\nu_{1}\right) \Gamma\left(\nu_{2}+1\right)} x(k, t)\right]^{2 n\left(\nu_{1}-\nu_{2}\right)}\right]
\end{aligned}
$$

where $-1 / 2<\nu_{1}<1 / 2$ and $1 / 2<\nu_{2}<3 / 2$.

Now we are interested in obtaining the spectrum of the $\psi$ and $\varphi$ squared-fluctuations. Their spectrums $\mathcal{P}_{\varphi}(k, t)$ and $\mathcal{P}_{\psi}(k, t)$ on cosmological scales, are given respectively by the expressions

$$
\begin{aligned}
\left.\mathcal{P}_{\varphi}(k, t)\right|_{I R} & =\frac{k^{3}}{2 \pi^{2}}\left(\tilde{\xi}_{k} \tilde{\xi}_{k}^{*}\right) \simeq \frac{k^{3}}{2 \pi^{2}}\left[A_{1}^{2}(t) k^{-2 \nu_{2}}+\left[B_{1}(t) k^{2\left(\nu_{2}-\nu_{1}\right)} \sum_{n=0}^{\infty} \frac{\left[\alpha_{1} x(k, t)\right]^{2 n\left(\nu_{2}-\nu_{1}\right)}}{\left(v_{1}+1\right)}\right.\right. \\
& \left.\left.+B_{2}(t) k^{2\left(\nu_{1}-\nu_{2}\right)} \sum_{n=0}^{\infty} \frac{\left[\alpha_{2} x(k, t)\right]^{2 n\left(\nu_{1}-\nu_{2}\right)}}{\left(v_{2}+1\right)}\right]^{2}\right] \\
\left.\mathcal{P}_{\psi}(k, t)\right|_{I R} & =\frac{k^{3}}{2 \pi^{2}}\left(\xi_{k} \xi_{k}^{*}\right) \simeq \frac{1}{4 \pi(p-1)} \Gamma\left(\frac{p+1}{2(p-1)}\right)^{2}\left[\frac{2(p-1) \beta}{\pi}\right]^{\frac{p+1}{p-1}} k^{3-\frac{p+1}{p-1}}
\end{aligned}
$$


where

$$
\begin{aligned}
& B_{1}(t)=\frac{\beta(3 p-2) \phi_{0} \Gamma\left(\nu_{2}+1\right) \Gamma\left(\frac{p+1}{2(p-1)}\right)[2 \beta(p-1)]^{\frac{p+1}{2(p-1)}+\nu_{2}-\nu_{1}}}{2 \pi^{\frac{p+1}{2(p-1)}} \Gamma\left(\nu_{1}+1\right)\left[\left(\nu_{2}-\nu_{1}\right)(p-1)\right]} t^{-\left(\nu_{1}+1\right)(1-p)}, \\
& B_{2}(t)=\frac{\beta(3 p-2) \phi_{0} \Gamma\left(\nu_{2}\right) \Gamma\left(\frac{p+1}{2(p-1)}\right)[2 \beta(p-1)]^{\frac{p+1}{2(p-1)}+2 \nu_{2}}}{2 \pi^{\frac{p+1}{2(p-1)}} \Gamma\left(\nu_{2}+1\right)\left[\left(\nu_{2}-\nu_{1}\right)(p-1)\right]} t^{-\left(2 \nu_{2}+1\right)(1-p)}, \\
& A=-\frac{B \Gamma\left(\nu_{2}\right) t^{\nu_{2}(p-1)+(1-3 p) / 2}}{\pi[2 \beta(p-1)]} \\
& \alpha_{1}=-\frac{\Gamma\left(\nu_{2}+1\right) \Gamma\left(\nu_{1}\right)}{\Gamma\left(\nu_{2}\right) \Gamma\left(\nu_{1}+1\right)} \\
& \alpha_{2}=-\frac{\Gamma\left(\nu_{1}+1\right) \Gamma\left(\nu_{2}\right)}{\Gamma\left(\nu_{2}\right) \Gamma\left(\nu_{2}+1\right)}, \\
& v_{1}=\frac{5 p-3+2 \nu_{1}(p-1)}{4\left(\nu_{2}-\nu_{1}\right)(1-p)} \\
& v_{2}=\frac{5 p-3+2 \nu_{2}(p-1)}{4\left(\nu_{2}-\nu_{1}\right)(p-1)} .
\end{aligned}
$$

Notice that for $\left.p \rightarrow \infty \mathcal{P}_{\psi}(k, t)\right|_{I R}$ goes as $k^{2}$. An interesting result for $\mathcal{P}_{\varphi}(k, t)$ is that it depends on $3 p<m^{2}=4\left(6 \pi G \phi_{0}^{2}-1\right)<p(2 p-1)$, and hence it is required that $p>2$. On the other hand, for sufficiently large $t$ the first term in (28) is dominant, so that

$$
\left.\mathcal{P}_{\varphi}(k, t)\right|_{I R \mid t \rightarrow \infty} \sim k^{3-2 \nu_{2}}
$$

which approaches to a scale invariant spectrum for $p \rightarrow \infty$.

\section{FINAL REMMARKS}

In this work we have studied an example of the formalism developed in [14], which is an extension of the well-known formalism for gauge-invariant scalar metric fluctuations during inflation. The formalism here examined is valid also for fluctuations with large amplitude, but the equations are very difficult to be solved due to the non-linearity of the Einstein and Lagrange equations. In the proposal here studied vector and tensor perturbations of the metric are neglected and the fluid is considered as irrotacional. Of course, the analysis is only valid in a cosmological context on super Hubble scales when the universe expands adiabatically. We have confirmated that, for small fluctuations the linear approximations give us the same dynamics that for the standard method. In this work we have illustrated

one example where the universe grows with a scale factor $a(t) \sim t^{p}$, (with $p \gg 1$ ). We found 
that, for very large $p$, at the end of inflation the spectrum $\left.\mathcal{P}_{\varphi}(k, t)\right|_{I R \mid t \rightarrow \infty}$ becomes scale invariant on cosmological scales, but $\left.\mathcal{P}_{\psi}(k, t)\right|_{I R \mid t \rightarrow \infty}$ goes as $k^{2}$. However, at the beginning of inflation it is not true, because the spectrum of the $\varphi$-fluctuations is altered by the modes of metric fluctuations $\tilde{\xi}_{k}(t)$. Furthermore $\langle E|\psi| E\rangle \sim 1 / a$, so that we conclude that at the end of inflation (and later), next order effects due to metric fluctuations on cosmological scales should be negligible.

\section{Acknowledgements}

The authors acknowledge UNMdP and CONICET (Argentina) for financial support.

[1] A. Guth, Phys. Rev. D23, 347 (1981);

A. D. Linde, Phys. Lett. B108, 389 (1982);

A. Albrecht and P. J. Steinhardt, Phys. Rev. Lett. 48, 1220 (1982).

[2] A. D. Linde, Phys. Lett. B129, 177 (1983).

[3] A. Berera, Phys. Rev. Lett. 75, 3218 (1995);

M. Bellini, Phys. Lett. B428, 31 (1998);

A. Berera, M. Gleiser and R. O. Ramos, Phys. Rev. Lett. 83, 264 (1999).

[4] M. D. Pollock, Nucl. Phys. B298: 673 (1988);

M. Mijic, Int. J. Mod. Phys. A6, 2685 (1991);

D. S. Salopek, J. R. Bond, Phys. Rev. D43, 1005 (1991);

S. Mollerach, S. Matarrese, A. Ortolan and F. Lucchin, Phys. Rev. D44, 1670 (1991);

J. M. Stewart, Class. Quant. Grav. 8, 909 (1991);

S. Habib, Phys. Rev. D46, 2408 (1992);

M. Bellini, H. Casini, R. Montemayor and P. Sisterna, Phys. Rev. D54, 7172 (1996).

[5] M. Bellini, Phys. Rev. D63: 123510 (2001);

M. Bellini, Phys. Rev. D64: 123508 (2001).

[6] G. Dvali and S. H. Tye, Phys. Lett. 450, 72 (1999).

[7] M. Bellini, Nucl. Phys. B660, 389 (2003);

J. E. Madriz Aguilar and M. Bellini, Phys. Lett. B619, 208 (2005); 
M. Anabitarte and M. Bellini, J. Math. Phys. 47: 042502 (2006).

[8] J. M. Bardeen, Phys. Rev. D22, 1882 (1980).

[9] V. F. Mukhanov, JETP Lett. 41, 493 (1985).

[10] W. L. Power and L. C. Percival, Proc. Roy. Soc. Lond. A456, 955 (2000).

[11] M. Bellini, Phys. Rev. D64: 043507 (2001).

[12] V. Acquaviva, N. Bartolo, S. Matarrese and A. Riotto, Nucl. Phys. B667, 119 (2003);

F. Finelli, G. Marozzi, G. P. Vacca and G. Venturi, Phys. Rev. D74: 083522 (2006);

K. Nakamura, Phys. Rev. D74: 101301 (2006).

[13] G. D'Amico, N. Bartolo, S. Matarrese and A. Riotto, JCAP 0801: 005 (2008).

[14] E. W. Kolb, S. Matarrese, A. Notari and A. Riotto, Mod. Phys. Lett. A20, 2705 (2005). 